\title{
Pensar 'teóricamente' la práctica de la enseñanza de la Historia
}

Thinking 'theoretically' teaching History practice

Ana Zavala*

\section{RESUMEN}

Este artículo propone presentar los modos en los cuales la práctica de la enseñanza de la historia es teorizada, desde fuera (por académicos fundamentalmente) y desde dentro (por los propios profesores). Cada una de estas formas de teorización apela a fundamentos distintos, así como evidencia fines diferentes para abocarse a ella. Me interesará la confrontación entre estos dos modos de teorización, en tanto ambos articulan de diferentes maneras la práctica (de la investigación o de la enseñanza) y la teoría (la de la práctica - de la investigación o de la enseñanza - o la relativa a distintos registros de lo real), lo cual implicará algunas reflexiones en torno los usos de algunos términos claves para el acercamiento a esta temática. La tesis del artículo apunta no a forzar la contradicción entre la teorización académica y la práctica sino por el contrario a sugerir un modo de pensar su complementariedad. Palabras clave: enseñanza de la historia; práctica de la enseñanza de la historia; la teoría práctica de la práctica de la enseñanza.

\begin{abstract}
This paper is concerned with the arrangement of the ways in which teaching history practice is theorised, by outsiders (mainly academic people) and insiders (the teachers themselves). Each of these theorisation ways is differently grounded, as their intentions to be concerned with. I will be interested in the confrontation between both theorisation ways because they use to articulate differently practice (research or teaching one) and theory (this concerned with practice - research or teaching one - or this concerned with the diversity of the real), which will imply some reflexions on the uses of some key-terms to the approach of this subject. The thesis of this paper does not point to aggravate the opposition between academic and practical theorisation but, on the contrary, to suggest some way to their complementariness.
\end{abstract}

Keywords: teaching history; teaching history practice; practical theory of teaching practice.

\footnotetext{
* Facultad de la Cultura, Instituto Universitario - Centro Latinoamericano de Economía Humana. Montevideo, Uruguay. azavala@claeh.edu.uy
} 


\section{LAS PRÁCTICAS AJENAS Y LAS PROPIAS}

Este artículo me brinda la oportunidad de actualizar y replantear algunos asuntos que he abordado ya en trabajos anteriores, ${ }^{1}$ y que están directamente relacionados con la articulación entre las perspectivas académicas respecto de la práctica de la enseñanza de la historia y lo que los propios profesores de historia pueden decir al respecto. Para eso me parece que son necesarias algunas precisiones en relación al uso de ciertos términos o expresiones en la medida en que aparecen significadas de maneras diversas en el contexto bibliográfico e incluso en el habla corriente.

En primer lugar me interesa abordar la manera en que enseñanza y práctica de la enseñanza aparecen en muchos textos como sinónimos cuando puede argumentarse que remiten a cuestiones diferentes. Es cierto que uno puede hablar de la enseñanza como la práctica de la enseñanza, obliterando el hecho de que se está refiriendo a una práctica. También es cierto que hablar por ejemplo de la enseñanza de la historia remite no solo a las prácticas de enseñanza, sino a las políticas institucionales, a los planes y programas, a los fines establecidos para su enseñanza, a las dimensiones curriculares, y también a asuntos tales como la evaluación, la ejercitación o las dificultades que pueda presentar para los estudiantes el aprendizaje de los conceptos o el tiempo histórico por ejemplo. Por otra parte, la práctica de la enseñanza de la historia aparece a menudo vinculada con la 'didáctica de la historia', lo cual contribuiría a delimitar con más precisión los campos semánticos. El problema es que, en muchos casos, los artículos referidos a la didáctica y a la enseñanza de la historia se ocupan de los mismos asuntos (incluyendo por ejemplo, problemas de aprendizaje, análisis de programas y manuales, actuales y pasados, etc.).

A los efectos de este artículo, práctica de la enseñanza de la historia remite exclusivamente a lo que los profesores hacen cuando enseñan historia, incluyendo no solo las instancias de aula sino todas las que les son solidarias, como la preparación de clases y cursos, el diseño de tareas de ejercitación y evaluación, fichas de trabajo, presentaciones PowerPoint, Prezi, etc. para uso en el aula, salidas didácticas, etc. Didáctica de la historia queda entonces restringido a la teoría de esa práctica de la enseñanza elaborada por quien la lleva a cabo, y de eso me ocuparé en la segunda parte de este trabajo. La diversidad 
de asuntos que pueden ser considerados como relativos a la enseñanza de la historia queda naturalmente por fuera de lo que abordaré aquí.

En segundo lugar haré algunas consideraciones respecto del uso del término historia. Me apoyaré en algunas precisiones hechas con gran justeza por Michel de Certeau (1993, p.35):

Ciertamente, en el uso ordinario el término historia con-nota a su vez a la ciencia y a su objeto - la explicación que se dice y la realidad que ya pasó o está pasando. Otros dominios no presentan la misma ambigüedad: el francés no confunde en una misma palabra la física y la naturaleza. La misma apelación de "historia" nos sugiere una situación de proximidad muy particular entre la operación científica y la realidad analizada. El primero de ambos aspectos formará nuestra entrada en materia por diversas razones: porque la densidad y la extensión de "lo real" sólo son designadas y afectadas en su sentido en un discurso; porque esta restricción en el empleo de la palabra "historia" da su correspondiente a la ciencia (la ciencia histórica) ... en fin, para no meternos en la selva virgen de la Historia, región de una "rica imprecisión" donde proliferan las ideologías y donde corremos el riesgo de perdernos para siempre.

Lo que me interesa destacar aquí - y dando un paso más sobre el planteo de Michel de Certeau - es no solo la articulación entre unos eventos y un discurso a la vez descriptivo e interpretativo respecto de los mismos - es decir, la historiografía - sino la articulación entre ese discurso (que de hecho son varios discursos en la medida en que posiblemente los profesores hayamos leído más de un autor respecto del tema de nuestra clase) y el discurso de la clase de... historia. Una primera precisión tiene que ver entonces con el hecho de que la clase de historia remite al pasado vía la historiografía que el profesor conoce, que es su verdadero punto de referencia. Una segunda precisión nos obliga a pensar en que nadie enseña todo lo que sabe y de la manera exacta en que está en los libros que leyó... porque de hecho difícilmente lo recuerde. A esto podríamos agregar que un mismo tema puede ser abordado por un mismo profesor de maneras muy diferentes en razón de las circunstancias (tiempo disponible, curso de primer o segundo ciclo, naturaleza del grupo, posición que ocupa el tema en el proyecto de curso, etc.). En conclusión, la historia en la práctica de la enseñanza de la historia puede referir a configuraciones discursivas bien diferentes entre sí, sin que por ello esté - de oficio - puesta en 
cuestión la fidelidad a algún patrón historiográfico (que podría estarlo, y por muchas razones, independientemente del nivel académico en el cual la práctica tenga lugar).

A los efectos de este artículo pues, historia en el contexto de la expresión práctica de la enseñanza de la historia remite a la versión que el profesor da a otros de su saber, un saber proveniente en su mayoría de su lectura de los libros de historia, pero también de la escucha de profesores o historiadores. No coincide necesariamente con todo lo que ha leído o escuchado, alguna parte de lo cual con seguridad ha olvidado, y tampoco coincide en el volumen informativo o en la jerarquización de eventos mencionados en la clase o en los materiales usados a los efectos. Las dimensiones causales y conceptuales son las que, seguramente en el seno de otra configuración discursiva, tendrían que mostrar el grado de fidelidad al trabajo de los historiadores de referencia.

Finalmente, será la polisemia a la que está ligado el término teoría la que me interesará. Por un lado es cierto que el lenguaje corriente asume la teoría a veces como algo aún no comprobado y por lo tanto se usa como sinónimo de hipótesis (no sabe bien, pero yo tengo una teoría...), o bien como un discurso vacío en el cual 'la práctica' es lo que cuenta (en teoría...pero...). Por otro lado está 'la teoría' en el sentido de teoría científica, indefectiblemente asociada a la investigación y al respaldo de un cierto proceder metodológico que valida sus resultados. En este sentido, las teorías describen, explican y en muchos casos predicen el funcionamiento del mundo. Finalmente, hay también teorías de la práctica, que organizan el trabajo humano y producen un sentido para él. Demás está decir, que las formas en que teorías científicas y teorías prácticas conviven, particularmente en el discurso académico, es por cierto un asunto complejo.

Por esta razón, este artículo intentará un gesto transversal a través de las teorías científicas, a veces también llamadas formales - de hecho alcanzaría con decirles 'académicamente producidas' - y sus diferentes relaciones con las diferentes teorías prácticas que están detrás de las distintas prácticas implicadas en la teorización de la práctica de la enseñanza. Está claro (Latour, 1996; Kristeva, 1969) que una teoría es el producto de una actividad llevada a cabo por alguien, sea que estudie el funcionamiento del universo o la manera en que algunos profesores dan clase de historia en un cierto lugar. Esta actividad que produce teorías en torno a otra práctica que no produce teorías sino que 
produce clases de historia nos obliga a pensarla también como una práctica, que puede ser teorizada tanto desde dentro como desde fuera (como es el caso de la práctica de la enseñanza, la de la historia o cualquier otra).

De hecho, no disponemos en el espacio público de teorías acerca de la práctica de investigar acerca de cómo los profesores dan clases de historia (incluyendo lo que piensan acerca de ello); de lo que sí disponemos es del fruto de esa actividad que no nos da acceso a las teorías que guiaron el trabajo de investigación. Por otra parte y en contraste, disponemos - y en varios registros - de los modos en que los profesores de historia desarrollan actividades de teorización en torno a su trabajo de enseñar historia, asumiendo como herramientas de análisis a conceptualizaciones provenientes de diversos campos de saber (que van desde la historiografía y la filosofía de la historia hasta la lingüística, la semiótica, el psicoanálisis y la filosofía de la acción, entre otros).

Este artículo abordará entonces en primera instancia el campo de la teorización de la práctica de la enseñanza de la historia por agentes externos a la misma, intentando ver separadamente -a los efectos del análisis - la práctica (de algunos sujetos) y el producto de esa práctica (lo que han escrito acerca de la práctica de la enseñanza de la historia de otros sujetos). En una segunda instancia considerará los modos de teorización que están vinculados directamente a la práctica de la enseñanza de la historia para los cuales el sujeto de la acción es siempre singular y el objeto es - siempre y por definición - lo que ha hecho en su clase en algún momento. Tal como he anunciado más arriba, el equilibrio entre estas dos miradas teorizantes no es sencillo, ni siquiera en el plano especulativo. La conclusión estará dedicada a considerar algunas posibilidades de pensar una dimensión armonizadora entre ambas actividades teorizantes en torno a la práctica de la enseñanza de la historia.

\section{LA PRÁCTICA DE TEORIZAR LA PRÁCTICA DEL OTRO}

Sin temor a equivocarme, siento que puedo afirmar que para la mayoría de los autores de teorías acerca de lo que los profesores de historia hacemos en clase - de hecho acerca de cómo es la enseñanza de la historia - su propia práctica es invisible y sobre todo resulta irrelevante a los efectos de la consideración de las conclusiones a las que han arribado. En sus textos existen ciertamente referencias de autoridad a un campo del saber, o incluso a una 
orientación, como por ejemplo el análisis de discurso, el constructivismo, la sociología del conocimiento o la filosofía marxista, habermasiana, etc. Parafraseando a Barthes (1987) se podría decir que en esos trabajos la ciencia 'se cuenta sola', sin preocuparse de dar señales del emisor, que de hecho es el que firma el trabajo y se hace responsable por él.

Sin embargo, detrás de cada palabra de esos textos hay - además de un autor o varios - muchas horas de trabajo y estudio, una bibliografía de recibo y una que ha quedado de lado, una opción metodológica y por supuesto una buena razón para implicarse en esa investigación. Para decirlo brevemente: no es posible que esos libros y artículos no sean el resultado de una práctica, o más bien de varias: la lectura, el estudio, la investigación, la escritura, la evaluación... De todas formas, creo que se puede argumentar a favor de una cierta intención de ser invisible en tanto sujeto de la acción a partir de la ausencia muy difundida de la primera persona del singular, persistentemente sustituida o por el nosotros (que a veces es uno solo) o por el se (que en realidad es un yo). Es cierto que esto forma parte de una tradición de escritura científica muy sólidamente arraigada, pero también es cierto que no es inocente. La situación es paradojal, en el sentido de que se analizan prácticas llenas de sujetos (profesores, alumnos...) y ninguna de las herramientas que sirve para acercarse a sus prácticas rebota sobre el investigador y le permite verse a sí mismo como sujeto de una práctica que investiga las prácticas de otros. Desde mi punto de vista, la paradoja sin embargo se resuelve algunas veces transformando a los singulares en plurales generales (los profesores de historia), otras a través del estudio de casos, lo que finalmente habilita a hablar de el profesor, para nombrarlos a todos o a una gran mayoría representativa.

De hecho, siguiendo el modelo podría - desde fuera - yo también teorizar la práctica de investigar la práctica de la enseñanza de la historia desde fuera, y sugerir unos motivos para hacerlo, unas intenciones para la acción, una relación entre lo esperado y lo que se pueda considerar como realmente acontecido, etc. Vería entonces esa práctica, de hecho sus resultados, a la luz de la teoría práctica que la ha guiado. Está claro sin embargo que cualquiera de los autores aludidos que leyera esto, además de considerarlo posiblemente un gesto irreverente e innecesario (¿por qué razón tomar como objeto de estudio precisamente esas prácticas?), se sentiría bastante poco identificado con las conclusiones del trabajo, o al menos con unas cuantas. En realidad, la 
conclusión más honesta a la que arribaría, respecto de las teorías que han guiado las prácticas que han dado como resultado esos trabajos es: cada uno sabrá por qué se ha comprometido con ese proyecto y lo que valen para él o para ella las conclusiones a las que ha llegado. Como veremos en la segunda parte de este artículo, no cortaríamos camino pidiéndoles que nos lo dijeran. De todas formas me queda la sensación de que, justamente, si tuvieran cierta herramientas para ver la práctica de la enseñanza como una práctica, entonces también verían su trabajo como la práctica de un sujeto de acción.

Queda entonces claro que se trata de una práctica (la de la investigación) que toma como objeto de estudio a otra práctica (la de la enseñanza de la historia), teniendo por resultado (un artículo, un libro, una ponencia, es decir, un texto) que teoriza su objeto en diversos modos, llegando en algunos casos a asumir que teoriza también la teoría práctica que ha guiado la práctica de la enseñanza (que es su objeto principal de estudio). Desde este punto de vista no podría argumentarse en contra del hecho de que en tanto alguien investiga la práctica de la enseñanza de la historia lo hace por alguna razón y para (deseando) producir algunos efectos en alguien. De hecho, los profesores de historia la enseñamos también por alguna razón y para (deseando) producir algunos efectos en alguien, por lo cual es seguro que nos encontraremos con una serie de herramientas de análisis - de las prácticas - que resultarían apropiadas en ambos casos.

En el marco de este artículo me atendré sin embargo no a las prácticas de investigación en torno a la práctica de la enseñanza de la historia - que me son tan esencialmente ajenas y opacas como las teorías prácticas que las han guiado - sino a sus resultados públicos y materiales: los textos. Para esto no solo tendré en cuenta que son el efecto de unas prácticas (estudio, lectura, investigación, escritura) sino también el hecho de que existe un autor de la interpretación de esos textos, que soy yo, que tiene unas determinadas herramientas de análisis, además de motivos, intenciones, etc. para embarcarse en la elaboración de este trabajo. En ese sentido, el uso del modo indicativo comparte siempre la pretensión descriptiva con la tarea de autor de configurar la realidad a través del lenguaje. ${ }^{2}$

Enunciaré entonces una serie de características que me parecen transversales a una bibliografía muy amplia y muy bien conocida y que devienen de un acercamiento a esa bibliografía a lo largo de un período que ronda las cuatro 
décadas. Me ocuparé primeramente de las formas en que la práctica de la enseñanza de la historia es entendida (es decir, teorizada) para luego abordar las formas en que la teorización práctica que guía la enseñanza - de la historia solo en algunos casos - es entendida por algunos autores.

En primer lugar, y en un gesto común con gran parte de la investigación académica, la práctica de la enseñanza de la historia convertida en objeto de estudio produce textos de naturaleza descriptiva (cómo) que son a su vez interpretativos (por qué razón). Es el caso, por ejemplo, de los trabajos de Henry Pluckrose, Suzanne Citron, Nicole Lautier, Henri Moniot, François Audigier, Nicole Tutiaux-Guillon, Pilar Maestro, Raimundo Cuesta, Joan Pagès, tomados como representativos de una lista mucho más larga. ${ }^{3}$ Estas descripciones no van por el lado de lo clínico, del trabajo en singular respecto de la práctica de la enseñanza de la historia, sino por el contrario se esfuerzan en todo momento por obtener conclusiones de validez generalizadora (en la mayoría de los casos a nivel nacional). Cualquiera de las citas que aparecen más abajo da cuenta de esta perspectiva generalizadora y homogeneizadora, en relación a la práctica de la enseñanza de la historia.

Dos aspectos me interesa remarcar en este punto: el primero de los cuales es que no siempre parece necesario tomar contacto directo con la práctica de la enseñanza. En efecto, en muchos casos el conocimiento de lo que los profesores han enseñado y la manera en que lo han hecho surge del análisis de los programas oficiales, de los manuales o de las producciones de los estudiantes. ${ }^{4}$ De esta manera es posible a menudo proponerse estudiar comparativamente los modos de enseñanza de la historia del presente y del pasado. Me interesa también remarcar el hecho de que es escasísima, por no decir nula, la bibliografía de corte académico respecto de la práctica de la enseñanza de la historia que considere que existen profesores que enseñan bien. Posiblemente la arista común a muchos estudios sea la vinculación del enfoque de la historia adoptado por los profesores, a menudo tenido por anticuado - es decir positivista o asimilable - o malamente simplificador del contenido historiográfico de referencia. Es esta mirada la que ha producido la idea de 'historia enseñada' para marcar ya sea una diferencia de calidad o una de naturaleza - o ambas - respecto de la producción historiográfica, como lo vemos en el siguiente ejemplo: 
La place des concepts, et plus encore la conceptualisation sont limités. Dans l’enseignement ordinaire, ils se confondent avec les réalités qu’ils désignent. Les mots définis sont peu nombreux ... et la définition est donnée par l'enseignant; ... 'je vais vous demander de faire votre propre définition', pour très vite passer à 'voilà la définition que je vous donne' ... Ce sont plutôt les termes aisément évaluables que les concepts fondamentaux qui son définis. Plutôt les objets que les mots pour le dire que si sont travaillés. (Tutiaux-Guillon, 2004, p.4)

En segundo lugar, y teniendo en cuenta las consideraciones anteriores, el perfil prescriptivo explícito o implícito constituye una nota común en los trabajos respecto de la práctica de la enseñanza de la historia. Vinculados a los fines, a la percepción de lo real, o a distintas teorías del aprendizaje (de donde surge por ejemplo que hay una manera 'constructivista' o 'positivista' de enseñar la historia), e incluso a la formación docente, muchos trabajos acaban proponiendo una manera de hacer las cosas bien, o al menos mejor de lo que se hace tan comúnmente como se ha visto en los trabajos de corte descriptivo. Tomo el caso de Pilar Maestro, entre muchos posibles:

El propósito de este escrito es ofrecer una reflexión sobre algunos elementos básicos en la enseñanza-aprendizaje de la Historia que nos sirvan para dibujar una propuesta alternativa desde las líneas de la investigación que nos parecen más interesantes. (Maestro, 1991, p.55, destacado mío)

Es de hacer notar que sin la idea de una matriz general respecto de la práctica de la enseñanza de la historia, la producción de trabajos de corte prescriptivo no tendría sentido. Pienso al mismo tiempo en trabajos dedicados a la enseñanza de temáticas específicas, como por ejemplo cómo enseñar el tiempo histórico:

La enseñanza que se basa en una concepción positivista de la historia da lugar en el alumnado a unos aprendizajes memorísticos o generados por ingenuas relaciones causa-efecto. Los esquemas de conocimiento que el alumnado construye sobre el tiempo están limitados a una determinada interpretación de la historia, y por tanto, el tiempo histórico queda relegado a una de sus partes, que es el tiempo cronológico, atrapada en una narración histórica que sólo ofrece respuestas y ninguna pregunta ... Desde este punto de partida, proponemos una deconstrucción del tiempo en el alumnado para reconstruir su conocimiento desde nuevos 
parámetros. Deconstruir el tiempo no quiere decir destruir los conocimientos históricos adquiridos, sino reinventar el tiempo en función de nuevas perspectivas para su aprendizaje. Deconstruir el tiempo histórico quiere decir poner en evidencia los convencionalismos que han generado determinadas visiones de la historia, presentar las contradicciones que estas visiones provocan, $y$, a la vez, ofrecer nuevos enfoques de comprensión de la temporalidad humana. (Pagès; Santisteban, 1999, p.195)

De alguna manera, la prescripción supone la posibilidad de incidir en las prácticas de los profesores, en general o individualmente considerados. Ha sido precisamente cuando los investigadores han percibido los límites de su influencia en el terreno de las prácticas que han aparecido distintos tipos de conceptualizaciones respecto de esta 'resistencia'. Mientras que para algunos autores tiene que ver con un problema de formación o de capacidad profesional y no se ocupan muy explícitamente de la cuestión, para otros es precisamente la existencia de algún tipo de teoría de la práctica lo que está detrás de este rechazo o desconocimiento.

En este punto da la impresión de que los trabajos provenientes del campo de las didácticas disciplinares insisten de formas más o menos veladas en la prescripción, mientras que autores ligados a un análisis más general de la profesión docente toman de otra manera el hecho de que los profesores actúen según sus propias teorías a la hora de dar clase. En relación a esta segunda postura se destacan los nombres 'fundadores' de Lawrence Stenhouse, Wilfred Carr y Donald Schön. De hecho son ellos quienes han introducido la idea de que los profesores somos los teóricos de nuestra propia práctica y de alguna manera han dado lineamientos para pensar en el asunto.

Sin embargo, y contrariamente a lo que podría pensare en la lógica natural de estas proposiciones, para estos autores las teorías de los profesores, las que guían sus prácticas, no son como las de los académicos. Son tenidas habitualmente por creencias (Stenhouse, 1983; 1988), y como tales dan cuenta de un trabajo intelectual de otra naturaleza (y de otra calidad) (Carr, 2006; 1990). Por su parte Schön y Argyris (1989) prácticamente las invalidan arrinconándolas en el lugar de las teorías profesadas, o sea falsas teorías que se apropian inadecuadamente del contenido de teorías científicas para dar cuenta de una práctica, de la que finalmente no dan cuenta. Es necesario superarlas y llegar a las (verdaderas) teorías en acto que ajustan perfectamente con la práctica teorizada. 
Para terminar, y retomando las consideraciones que he hecho anteriormente respecto de las conclusiones referentes a la calidad de las prácticas de enseñanza de la historia, es posible llegar a la conclusión de que esta bibliografía es funcional a una configuración del rol docente como necesariamente dependiente de las directivas de la academia. Lo es, como hemos visto, tanto se ocupe de la descripción y análisis de las prácticas de enseñanza - más aún si derivan en prescripciones para la enseñanza o para la formación docente -, o que lo haga en relación a la teorización práctica que guía las prácticas de los profesores.

Posiblemente incrustadas en una tradición varias veces milenaria que separa la teoría de la práctica, no solo por su naturaleza sino por la relación jerárquica existente entre ellas, las formas en que unos se abocan a teorizar la práctica de otros hacen que esos modos de teorización queden, a mi manera de ver, encerradas en un círculo vicioso que les permite sobrevivir 'del otro lado del río', invisibles, opacas, inasibles... Para que esto suceda, la dimensión ajena del objeto de estudio - o sea, los modos en que los profesores teorizan sus prácticas - ha de quedar legitimada por la configuración de una condición subalterna (Spivak, 1998) que lo vuelve definible a la vez que da sentido al trabajo sobre el otro como si en lugar de ser sujeto fuera objeto. En este sentido podemos recorrer a lo largo y a lo ancho la bibliografía que teoriza de muy distintas maneras las prácticas de la enseñanza de la historia y allí encontraremos la voz de los profesores invariablemente interpretada, es decir, diciendo lo que quiere decir lo que han dicho en la entrevista, o en la encuesta, o aún en la clase observada. Para eso, desde mi punto de vista, es casi necesario que la historia se enseñe mal, de forma deficiente, mejorable, porque tal vez si las clases fueran buenas, incluso maravillosas, es posible que la energía no brotara de ningún lado. Lo mismo para el caso de que las teorías de los profesores fueran realmente buenas, apropiadas, sensatas...

Imposible no pensar, desde fuera, cuál es el sentido de embarcarse en una empresa que se permite - de tanto en tanto - reconocer que no logra incidir en la configuración o en la mejora de las prácticas de enseñanza. Tal vez ese no sea el 'verdadero' sentido, tal vez sea simplemente una teoría profesada funcional a una situación de interlocución, como las de tantos practicantes que comunican a terceros el sentido de sus prácticas. 
Hemos llegado pues a un punto en el cual lo que importa es que hay dos prácticas: la analizada y la que lo analiza. Hay también, y por supuesto, dos teorías prácticas que guían esas prácticas, cuyo contenido como veremos es siempre menos interesante que el hecho de su propia existencia (cf. Ferry, 1990, p.84-85).

\section{LA PRÁCTICA DE TEORIZAR SU PROPIA PRÁCTICA}

Cruzamos pues la frontera y nos adentramos en un terreno en el que lo que es para otros objeto de análisis deja de serlo. A mismo tiempo, ponemos un pie en el - para muchos - inabordable pantano de la singularidad. Ya sea que alguien dé una clase de historia o que la analice a partir de la observación o de otros elementos, esa práctica es una combinación singular y única de elementos que configuran sus motivos, sus intereses, lo que se 'invierte' en ella, lo que se espera hacer y también lo que se espera/desea que influya en otros... Es en este punto que la referencia a un abordaje clínico se hace verdaderamente pertinente.

Lo primero que me interesa abordar es la cuestión de la relación entre la acción de un sujeto y su comprensión por parte del mismo, porque esta es la base de la actividad teorizante de la práctica de la enseñanza. En este sentido no podremos ir al fondo del asunto si consideramos la comprensión (de un evento, de mi propia acción o de un texto leído) como un estado firme, estable e inmutable. Esto no quiere decir que, como una veleta, la comprensión gire hacia un lado y hacia el otro según el viento, sino más bien que no hay que pensar que la forma en que comprendo esto en este momento tenga que ser necesariamente la misma en que lo comprenderé para siempre. De alguna manera posturas 'criticas' como la de W. Carr o la de D. Schön apuestan justamente a un cambio en la comprensión que el sujeto tiene de su propia acción. Sin embargo, desde su punto de vista existe una comprensión 'de baja calidad' que ha de ser sustituida - mediante la reflexión fundamentalmente - por otra 'genuina'. Visto desde dentro, es posible que una nueva comprensión represente un viraje importante respecto de la anterior, pero también es posible que lo que haga sea profundizarla y mantenerla recurriendo a herramientas de análisis más sofisticadas. 
Esto nos lleva directamente al lugar del lenguaje, formando parte de la acción y de su comprensión. Si se trata de una clase - y más si es una de historia - el lenguaje se vuelve un asunto omnipresente. Lo es porque la clase está hecha de lenguaje en mucho de lo que sucede en ella, los profesores y los alumnos se comunican a través del lenguaje, pero además, lo que es el centro de esta comunicación es el conocimiento histórico, que está escrito en los libros, en los manuales o en los textos que hay para leer en clase. Por otra parte, cualquier acto de previsión de la clase (plan de curso, de clase, guión, proyecto, etc.) está expresado en palabras, de la misma manera que no hay análisis, reflexión, teorización si no es justamente mediante el uso del lenguaje. Que se trate de un lenguaje formal, sintácticamente organizado y comunicado a otros, o que sean formas predicativas, internas, privadas, íntimas... es el lenguaje el que da forma al pensamiento. Desde este punto de vista, el primer nivel de teorización de la práctica se encuadra en la manera en que cada pequeño tramo de la acción (en el aula o fuera de ella) tiene sentido. Ricœur lo ha llamado semántica de la acción $n^{5}$ dando cuenta precisamente de la relación entre el lenguaje cotidiano y la acción humana. Está claro que ese sentido es siempre una construcción personal (Barbier, 2000) que articula necesariamente un tiempo pasado, su propio presente y diversas dimensiones de futuro (lo que haré en la próxima clase, qué propondré a los estudiantes el día de las pruebas, lo que imagino que comprenderán/aprenderán/valorarán los estudiantes, lo que ya mismo me estoy dando cuenta de que tengo que cambiar o que por el contrario, me encanta).

Por otra parte, esta vinculación con el lenguaje se articula con la relación con el saber (Beillerot, 2000) del profesor, que es idiosincrásica, afectiva, autobiográfica, ideológica... es decir, identitaria. Es cierto que todos somos profesores de historia, y muchos hemos leído los mismos libros y hasta hemos sido compañeros de clase, pero también lo es que cada uno tiene una relación con la historia, con el tema, con la orientación, con el historiador y con lo que cree que es mejor ofrecer a los estudiantes en esa clase, que siempre tiene algo en común con algún otro, y algo diferente, parecido o totalmente opuesto. Desde este punto de vista, hablar de 'la historia' que enseñan los profesores de historia es ciertamente aventurado, pues es posible que 'la historia' sea algo diferente en cada caso. Obviamente no estoy pensando en que esto implique que para unos los romanos hayan vencido en Cannas y para otros hayan sido vencidos por los cartagineses. Más bien estoy pensando en las maneras en que el 
discurso de la clase (incluyendo las partes expositivas, los dispositivos auxiliares como pizarrón, fichas de trabajo, propuestas de ejercitación, etc.) reconfigura necesariamente el discurso la historiografía de base en el recorte de la información, en la configuración conceptual y en los entrelazamientos causales. Es posible que las razones que están detrás de los modos en que el discurso de (una parte) la historiografía (que es la que el profesor conoce, recuerda y juzga pertinente tener en cuenta en ese momento) pasa al discurso de su clase constituya el centro de su teoría de la práctica de la enseñanza de la historia. Este gesto está inscrito en la historia personal y puede unas veces ligarse con el estudiante que cada uno fue, con las temáticas/orientaciones más o menos 'preferidas', con los desempeños escolares, con la relación - modelizante o todo lo contrario - con ciertos docentes...

Está claro entonces que es actuando que el sujeto de la acción concede un sentido a esa acción, sentido que a veces está apoyado por un plan previo, pero esto no es necesario en cada momento de una acción como la de enseñar en un aula, que está plagada de eventos inesperados. Está igualmente claro que en el mundo profesional, no solo el de los profesores, analizar y teorizar lo que se ha hecho representa en muchos casos un beneficio adicional. No debemos sin embargo confundir la acción de enseñar en el aula con la de teorizar esa práctica en otro momento, por otras razones y con otras expectativas. Se trata definitivamente de dos acciones diferentes, la segunda de las cuales no podrá sin embargo abordar separadamente la práctica (es decir, la acción de enseñar) de sus respaldos 'teóricos' (es decir del sentido producido en función de los motivos, las expectativas y las dimensiones afectivas presentes en la acción analizada). Es lo mismo si se trata de teorizar la práctica de la investigación o la de la gestión educativa.

Desde este punto de vista podemos encontrar una similitud y una diferencia radical con las situaciones planteadas en la primera parte del artículo. Existe, en ambos casos, una acción analítica, teorizante en torno a otra acción, o a otras acciones. Un investigador, o un profesor, analizan una acción de enseñanza de la historia. La diferencia radical está en el hecho de que en el segundo caso coinciden el actor de la acción analizada y quien se aboca a la tarea de analizarla. Esto nos lleva a tener en cuenta que aunque se trate en ambos casos de un análisis de una práctica, las razones por las cuales se hace y lo que se espera que surja de esa actividad analítica no tienen el mismo perfil. 
La idea de interrogar un objeto 'opaco' para comprenderlo que es de recibo en la investigación académica, no lo es tanto en la teorización práctica. De hecho es difícil pensar que un profesor da una clase de historia sin comprender lo que ha hecho, cosa que logra hacer a posteriori y bajo la tutela de 'expertos'. Lo que para algunos es difícil de entender es por qué, si ya lo entendió, insiste en una tarea de 'sobreteorización' de esa práctica y asiste a un taller de análisis de las prácticas de enseñanza. Como es de esperar, no puede haber una respuesta universal a esta pregunta. Tal vez solo anotar que hay algunos profesores, que por algunas razones se suman a estas empresas, y otros que, por otras razones - aunque sepan de su existencia y estén en condiciones materiales de asistir - no lo hacen. De todas formas, que sus procesos de análisis de su práctica no sean comunicados a otros no significa que no existan. Muchas veces tenemos noticia de ellos ex post facto: 'Estuve pensando y no sé por qué siempre... así que decidí que.... Finalmente, es cierto que mientras los destinatarios de la teorización académica son otros, que la leerán, valorarán y legitimarán, en el caso de los profesores los destinatarios de su trabajo son ellos mismos, singularmente considerados.

La tarea de análisis de la práctica descansa sobre la reconstrucción discursiva de la acción a analizar, hecho que puede ser común con otro tipo de análisis como hemos visto anteriormente. Aparte del hecho de que no tiene el mismo sentido relatar una clase para una persona que quiere saber cómo fue (y tal vez a uno no le interesa mucho decirlo, justo ahora), que hacerlo porque uno siente que será provechoso, interesante, etc., hay que tener en cuenta además un par de detalles: el primero es que el autor del relato y quien hace el análisis es la misma persona, de forma que esta puesta en palabras puede continuamente enriquecerse e incluso ser relativamente 'sintética' a cuenta de todo lo demás que obra en su mente y puede recuperar en cualquier momento. El segundo es que ese relato está normalmente dirigido a colegas que están en condiciones de hacerse cargo del sentido de frases o expresiones que desde fuera pueden resultar relativamente enigmáticas: 'después de hablar de las consecuencias de la crisis empecé a hacer un cuadro en el pizarrón’. Está claro que 'las consecuencias de la crisis' no representan un problema de comprensión para quienes escuchan o leen el relato, así como que el autor del relato quiere hacer foco en otra cosa y por lo tanto no considera relevante hablar del descenso de la población, del alza del costo de vida, o de otras circunstancias a las 
que la crisis dio lugar como lo hizo dentro del aula. La reconstrucción discursiva de una clase no es su transcripción textual, como si fuera el mapa de Borges. Sin embargo, puede por momentos transcribir lo más fielmente posible un diálogo, una pregunta, un comentario... o las consecuencias de la crisis si de eso se trata la cuestión a analizar.

Esto quiere decir que el foco del análisis es siempre cuestión de la mirada y la intención del analista, que en este caso es el mismo que el analizado. Por esta razón el fruto del análisis tiene el color de las herramientas de análisis movilizadas, que necesariamente hacen juego con el interés del analista en hacer foco en una determinada cuestión de su clase de ayer, su enfoque de un cierto tema o su manera de enfrentar un cierto tipo de situaciones. No se trata nunca pues de acomodar el análisis a las herramientas sino por el contrario de buscarlas en función del espíritu del análisis (es decir, teniendo en cuenta las razones por las cuales se hace y qué se espera de él). ${ }^{6}$ Conviene siempre tener en cuenta - para todos los casos - que el resultado del análisis tiene siempre que ver con qué herramientas se utilizaron (y con las que fueron dejadas de lado por las razones que sea).

En el análisis de una secuencia de clase se puede movilizar la historiografía de referencia e incluso la filosofía de la historia como herramientas de análisis. Si el enfoque de un tema sigue más o menos fielmente a un historiador, o si el profesor ha intentado una integración de dos visiones, o si el diálogo entre ambas ha sido el eje de la propuesta del tema, volver a los historiadores parece ineludible. Y si es el caso, la postura historiográfica o filosófica del autor o de los autores aludidos vendrá también en auxilio del análisis de la temática de la que se ha ocupado la clase. Es posible sin embargo que el foco del análisis esté en el diálogo con los estudiantes lo que llevará las líneas de análisis en muchas direcciones, que pueden ir desde miradas cognitivas, psicoanalíticas, de análisis del discurso, semióticas, etc. A veces, el punto son los afectos, los desencuentros, las frustraciones, los pequeños placeres... y entonces serán otras las herramientas convocadas.

Es de hacer notar que el análisis de una clase - ya sea que guarde similitud o distancia - se apoya en infinidad de casos en su propio proyecto. Es habitualmente el guión del curso, o aún su proyecto, el que organiza la trama general del relato y también la dimensión valorativa de lo que ha sucedido. No es que si una clase se parece ampliamente a su guión eso la haga 'mejor', a veces es 
justamente el apartarse del guión lo que hace de lo acontecido algo interesante. Dicho sea de paso, hacer un guión o preparar los materiales que serán necesarios para la clase de mañana es también una acción distinta de esa clase, y en tanto tal, tiene sus propios motivos, razones para existir y expectativas respecto de cómo incidirá en el futuro que se está representando anticipadamente.

De todas formas, el relato de la clase, como la clase misma, tienen mucho que ver con lo que el profesor previó hacer durante su desarrollo, que a veces es sensiblemente diferente a lo que demandan algunas formalidades administrativas. También tienen mucho que ver con todo lo que no fue nuevo en ese momento, lo que forma parte de la experiencia del sujeto, que muchas veces traza líneas larguísimas en el tiempo anterior. No hay forma de que este análisis no tenga un signo identitario, ya sea que lo reafirme, ya sea que contribuya a forjarlo.

Para terminar, me interesa destacar la singularidad de un momento de teorización práctica respecto de la práctica de la enseñanza. Esta singularidad está antes que nada anclada en que se trata de un profesor que se aboca a repensar los fundamentos de una acción de enseñanza que ya ha acontecido. Es singular porque es un profesor y porque es una práctica, pero también lo es porque la forma en que se entiende en ese momento lo que pasó en la clase (o lo que sea que se esté analizando). La próxima vez, si existe, que ese asunto sea puesto en análisis, será diferente. Esto no quiere decir que su autor se desdiga, aunque puede hacerlo y rotar $180^{\circ}$ en el punto de mira, y ver negativamente lo que antes fue positivo, etc.; lo que quiere decir que el nuevo momento de análisis no ignora el anterior. En los hechos, es muy frecuente que un análisis atraviese varios textos luego del primero, que es esencialmente narrativo y descriptivo de lo acontecido (aunque no puede no ser interpretativo en sí mismo), y por lo tanto se vaya configurando progresivamente, avanzando, renovándose, abandonando conclusiones iniciales...

Por otra parte, esta dimensión singular está siempre incrustada en un curso de acción, en el que se inscriben acciones pasadas (en la enseñanza, en ese curso, con ese grupo o con otros, con ese tema, con esa modalidad de abordaje, enfrentando ese tipo de problemáticas, etc.) y que además esperan el advenimiento de acciones futuras (la clase de mañana, el año que viene, el mismo tema en otro grupo, la misma situación con estos o con otros estudiantes). El trabajo de teorización está así incorporado y entrelazado con la 
dimensión práctica de la enseñanza de la historia. Me gustaría agregar que no hay profesores que teoricen y otros que no lo hagan, o unos que lo hagan mejor y otros peor. El prestigio del término 'teoría' tiende a hacernos pensar que la actividad teorizante en relación a las prácticas es un indicativo de superioridad o de destaque de algunos profesionales. De hecho, todas las prácticas están necesariamente acompañadas de una dimensión teorizante, con la cual a veces desde fuera acordamos y a veces no, pero eso no determina ni la existencia de una teoría práctica, ni el valor de la misma (que es necesariamente una atribución discursiva).

Tenemos que pensar que el acceso a las teorías prácticas por parte de terceros es una aventura compleja e incierta, en tanto aquellos a quienes les son comunicadas acaban formando parte del discurso porque forman parte del contexto de interlocución. También tenemos que pensar que la comprensión de su práctica por parte del sujeto de la acción no es una fórmula estable, y que el propio devenir de la práctica o la renovación de sus herramientas de análisis renueva - en poco o en mucho - lo que hoy piensa de lo que hizo ayer en esa clase de su curso de primer año. Es por esta razón que he planteado más arriba que lo que resulta más interesante, y con potenciales consecuencias para el entendimiento de la naturaleza de las prácticas docentes y sus anclajes en teorías prácticas, es más bien la existencia de esas teorías prácticas y no un contenido al cual - además de ser inestable - es difícil de acceder.

\section{CONCLUSIÓN: UN DIFÍCIL EQUILIBRIO}

La apuesta principal de este artículo, tal vez en contra de las hipótesis que puedan surgir de su primera lectura, es que la diferencia entre las teorizaciones respecto de la práctica de la enseñanza de la historia venidas desde dentro y desde fuera de esa práctica es simplemente un dato de la realidad. No son lo mismo, pero eso no quiere decir que haya que suprimir una en beneficio de la otra, o de que esta dualidad implique para cada una un cuestionamiento por parte de la otra.

Las formas en que la academia intenta comprender el mundo de la enseñanza, dentro del cual está el de las aulas, al cual pertenece la práctica de la enseñanza de la historia, forman parte de un gesto fundacional de la creación de saberes que nos acompaña desde hace largo tiempo. Que los saberes 
producidos estén acompañados en alguna medida del deseo, al menos de la intención de cambiar el mundo - que para el caso es su objeto de estudio - es también un gesto compartido por muchos campos de saber. Sin embargo es cierto que cuando se trata de oficios de lo humano como los llama Mireille Cifali (2005, p.19-21), donde el objeto es la práctica de otro sujeto la situación no es la misma que cuando el objeto son animales o fenómenos de la naturaleza, incluso fenómenos sociales, políticos, económicos o culturales, por llamarlos de alguna manera, y que incluyen naturalmente a la historia.

De alguna manera el acercamiento al objeto práctica ha permitido ver la existencia de las teorías prácticas que la acompañan de forma ineludible. Ha sido entonces que algunos autores (Ferry, Carr, Stanhouse, Elliott) han elevado a los profesores a la categoría de teóricos y de investigadores en un gesto inacabado en la medida en que con la otra mano los hace 'de segunda'7 montando un escenario de discordia, oposición y recelo, como el que vemos, por ejemplo, en Raimundo Cuesta:

Resulta proverbial el rechazo de los profesores de Bachillerato (entre otros del gremio docente) a las teorías que persiguen explicar o guiar su práctica. Se trata de una profesión profundamente apegada al prejuicio que idealiza la acción en el aula como principal y casi exclusiva referencia profesional. Detrás de ello se ampara una debilidad teórica corporativa muy acusada, que propende a considerar "retórica legitimadora" de gentes ajenas a la enseñanza toda reflexión allende la práctica inmediata. (Cuesta, 1998, p.168, destacado mío)

Desde mi punto de vista, la lógica que acepta la existencia de la teorización práctica, esa que sólo los practicantes (en este caso los profesores de historia) pueden hacer está disponible a partir del aparato teórico producido por la propia academia, que tiene cada vez menos inconveniente en reconocer que sus propuestas para guiar o mejorar la acción de enseñanza tienen cada vez más dificultad en concretarse. Las teorías de la acción, la filosofía del lenguaje, el psicoanálisis, la psicología cognitiva, entre otros, permiten perfectamente fundamentar la existencia y el funcionamiento de una teoría guiando la práctica, elaborada por el propio practicante, profesor o investigador. Lo que falta, a mi manera de ver, es unir las dos partes y actuar en consecuencia.

Tal vez pensando que sin el trabajo teorizador de la academia las teorías prácticas quedarían encerradas en el círculo del lenguaje común - que no es 
poca cosa pero tampoco es suficiente para darle vuelo y profundidad a la actividad teorizante de los profesores - podemos valorar la importancia del trabajo académico. Insisto, una vez más, en la centralidad de las herramientas de análisis en toda la empresa teorizadora de las prácticas de la enseñanza (y obviamente también en las de la investigación). Su producción y su utilización - de hecho convertir saberes y puntos de vista en herramientas de análisis - es posiblemente la empresa que convoca por igual a académicos (de fuera, y no solo de ciencias de la educación o de didáctica) y a profesores (necesariamente de dentro) habilitando con toda seguridad no una nueva manera de hacer las cosas, sino de entenderlas - se hagan como se hagan, por las razones que sea. La idea de mejorar de las prácticas también tendrá otra(s) cara(s), como se ve a menudo en el inframundo clínico de las prácticas cotidianas.

\section{REFERENCIAS}

ARGYRIS, Chris; SCHÖN, Donald. Theory in practice: Increasing Professional Effectiveness. San Francisco/London: Josey-Bass, 1989.

AUDIGIER, François. Didactique de l'histoire, de la géographie et des sciences sociales: propos introductifs. Revue Française de Pédagogie, n.85, p.5-10, oct.-nov.-déc. 1988.

BARBIER, Jean-Marie. Rapport établi, sens construit, signification donnée. In: .; GALATANU, Olga (Ed.) Signification, sens, formation. Paris: PUF, 2000. p.61-86. . Vocabulaires d'analyse des activités. Paris: PUF, 2011.

BARTHES, Roland. El discurso de la historia. In: El susurro del lenguaje, más allá de la palabra y la escritura. Barcelona/Buenos Aires: Paidós, 1987. p.173177.

BEILLEROT, Jacky. Le rapport au savoir. In: BEILLEROT, Jacky et al. Formes et formations $d u$ rapport au savoir. Paris: L'Harmattan, 2000. p.39-57.

BERNER, Christian; TOUARD, Denis (Ed.) Sens et interprétation: pour une introduction à l'herméneutique. Lille: Presses Universitaires du Septentrion, 2008.

CARR, Wilfred. Education without theory. British Journal of Educational Studies, vol. 54, no. 2, p.136-159, June 2006.

CARR, Wilfred. Hacia una ciencia crítica de la educación. Madrid: Laertes, 1990.

CIFALI, Mireille. Un métier de l'humain: une affaire de personne et de relation avec ses enjeux, ses exigences, ses peurs, ses pouvoirs. Nouvel Éducateur, n.172, p.1921, Oct. 2005. 
CITRON, Suzanne. L'histoire de France autrement. Paris: Éd. Ouvrières, 1992. Le Mythe national: L'histoire de France en question. Paris: Éd. Ouvrières, 1971.

COOPER, Hilary. Didáctica de la historia en la educación infantil y primaria. Madrid: Morata, 2002.

CUESTA, Raimundo. Clío en las aulas: la enseñanza de la historia en España, entre reformas e ilusiones. Madrid: Akal, 1998.

DE CERTEAU, Michel. La escritura de la historia. México: Universidad Iberoamericana, 1993.

FERRY, Gilles. El trayecto de formación: los enseñantes entre la teoría y la práctica. Barcelona: Paidós, 1990. p.84-85.

HEIDEGGER, Martin. Interpretaciones fenomenológicas sobre Aristóteles. [1922]. (Indicación de la situación hermenéutica) [Informe Natorp]. Madrid: Trotta, 2002.

KRISTEVA, Julia. Sémioké: recherches pour une sémanalyse. Paris: Seuil, 1969.

LATOUR, Bruno. Sur la pratique des théoriciens. In: BARBIER, J.-M. (Ed.) Savoirs théoriques et savoirs d'action. Paris: PUF, 1996. p.131-146.

LAUTIER, Nicole. Enseigner l'histoire au lycée. Paris: Armand Colin, 1997.

. L'appropriation de connaissances historiques et politiques par les adolescents. In: MONIOT, Henri; SEWANDKY, Maciej. L'histoire et ses fonctions: une pensé et des paratiques au présent. Paris: L'Harmattan, 2000. p.127-147.

LEE, Peter; SCHEMILT, Denis. Progression in understanding about historical accounts. Teaching History, n.117, p.25-31, 2004.

MAESTRO, Pilar. Historiografía, didáctica y enseñanza de la historia. La concepción de la historia enseñada. Clío y Asociados, n.2, p.9-34, 1997.

. Procedimientos vs metodología. Los procedimientos desde la disciplina y la metodología didáctica. IBER - Didáctica de las ciencias sociales, geografía e historia, n.1, p.53-71, 1994.

. Una nueva concepción del aprendizaje de la historia: el marco teórico y las investigaciones empíricas. Separata de: Studia Paedagogica (Revista de Ciencias de la Educación), Universidad de Salamanca, I.U.C.E., n.23, p.55-80, ene./dic. 1991.

MONIOT, Henri (Ed.) Enseigner l'histoire: des manuels à la mémoire. Berna: Peter Lang, 1984.

PAGÈS, Joan. El lugar de la memoria en la enseñanza de la historia. IBER - Didáctica de las ciencias sociales, geografía e historia, n.55, p.43-53, 2008.

.; SANTISTEBAN, Antoni. La enseñanza del tiempo histórico: una propuesta para superar viejos problemas. In: SIMPOSIUM DE DIDÁCTICA DE LAS CIENCIAS SOCIALES, X., 1999. Asociación Universitaria del Profesorado de Didáctica 
de las CCSS: Un currículum de Ciencias Sociales para el siglo XXI. Diada/Universidad de La Rioja, 1999. p.187-207.

PLUCKROSE, Henry. Enseñanza y aprendizaje de la historia. Madrid: Morata, 1993.

RICCEUR, Paul. Tiempo y narración I. Configuración del tiempo en el relato histórico. 5.ed. Madrid: Siglo XXI, 2004. p.113-166.

.; TIFFENAU, Dorian. La sémantique de l'action. Paris: CNRS, 1977.

SPIVAK, Gayatry Ch. ¿Puede hablar el subalterno? Orbis Tertius, La Plata: Centro de Estudios de Teoría y Crítica Literaria. Facultad de Humanidades y Ciencias de la Educación - Universidad Nacional de La Plata, Argentina, año III, n.6, 1998.

STENHOUSE, L. Artistry and teaching. The teacher as focus of research and development. Journal of Curriculum and Supervision, vol. 4, no. 1, p.43-51, fall 1988.

. The relevance of practice to theory. Theory into practice, vol. 22, issue 3 , 1983. Special Issue: Curriculum change/promised and practice, p.211-215.

TUTIAUX-GUILLON, Nicole. L'histoire enseignée entre coutume disciplinaire et formation de la conscience historique: l'exemple français. In: .; NOURRISSON, Didier. Identités, mémoires, conscience historique. Saint-Étienne: Presses Universitaires Saint-Étienne, 2003. p.27-41.

. Les conceptions de l'apprentissage auxquelles se réfèrent les enseignants seraient-elles un facteur d'inertie disciplinaire? In: JOURNÉES D'ÉTUDES DE DIDACTIQUES DE LA GÉOGRAPHIE ET DE L'HISTOIRE (JED 2004, Caen). Disponible en: http://ecehg.inrp.fr; Acceso en: 20 jul. 2015.

VERGNAUD, Gérard. Au fond de l'action, la conceptualisation. In: BARBIER, Jean-Marie (Ed.) Savoirs théoriques et savoirs d'action. Paris: PUF, 2000. p.275-291.

ZAVALA, Ana. Caminar sobre los dos pies. Didáctica, epistemología y práctica de la enseñanza. Práxis Educativa, Ponta Grossa, Brasil, v.1, n.2, p.87-106, jul./dez. 2006.

. Enseñamos, narramos, teorizamos. Introducción a: .; SCOTTI, Magdalena (Ed.) Historias de la enseñanza de la historia. Relatos que son... teorías. Montevideo: CLAEH, 2005. p.11-36.

. Mi clase de historia bajo la lupa: por un abordaje clínico de la práctica de la enseñanza. Montevideo: Trilce, 2012.

\section{NOTAS}

${ }^{1}$ Entre otros: ZAVALA, 2005; 2006; 2012.

${ }^{2}$ Ver por ejemplo: RICCEUR, 2004. Ver también: BERNER; TOUARD, 2008. 
${ }^{3}$ Presento a continuación una pequeña selección de obras de los mencionados autores que podría ampliarse sin ninguna dificultad incluyendo además, otros autores: PLUCKROSE, 1993; COOPER, 2002; CITRON, 1992; 1971; LAUTIER, 1997; MONIOT, 1984; AUDIGIER, 1988; TUTIAUX-GUILLON, 2003; MAESTRO, 1997; 1994; CUESTA, 1998; PAGÈS, 2008.

${ }^{4}$ Ver por ejemplo: LAUTIER, 2000; LEE; SCHEMILT, 2004.

${ }^{5}$ Ver por ejemplo: RICCEUR; TIFFENAU, 1977. También se puede ver BARBIER, 2011, particularmente las entradas : 'Lexique d'action' y 'Lexique de l'intelligibilité des actions', p.86-89; y VERGNAUD, 2000.

6 "La situación de la interpretación, en cuanto apropiación comprensiva del pasado, es siempre la situación de un presente viviente" y continúa: "El pasado solo se manifiesta con arreglo a la resolución y a la capacidad de apertura de la que dispone el presente" para concluir que es precisamente la explicitación de las herramientas de análisis lo que hace valiosa una interpretación, en tanto tal porque es "la condición fundamental que permite dar expresión al pasado en general" (HEIDEGGER, [1922]2002, p.30).

${ }^{7}$ CARR (1990, p.73) por ejemplo plantea: "Estas creencias [es decir, las teorías de los practicantes] pueden ser más o menos coherentes y sistemáticas, y cuanto más coherentes y sistemáticas sean, más se parecerán a una 'teoría”' (destacado mío).

Artigo recebido em 9 de agosto de 2015. Aprovado em 15 de outubro de 2015. 